\title{
StudyPlay: Um Modelo Gamificado Para Incentivo à Realização de Atividades Extraclasse
}

\author{
Aline Zanin ${ }^{1,2}$, Adalto S. Sparremberger ${ }^{2}$, Taila P. Becker ${ }^{2}$, Jorge L. V. Barbosa ${ }^{3}$ \\ ${ }^{1}$ Pontifícia Universidade Católica do Rio Grande do Sul (PUCRS) \\ Programa de Pós-Graduação em Ciência da Computação - PPGCC \\ ${ }^{2}$ Serviço Nacional de Aprendizagem Comercial (Senac-RS) \\ ${ }^{3}$ Universidade do Vale do Rio dos Sinos (UNISINOS) \\ Programa de Pós-Graduação em Computação Aplicada - PPGCA \\ \{azanin, assparremberger, tpbecker\}esenacrs.com.br, jbarbosa@unisinos.br
}

\begin{abstract}
The achievement of activities outside the classroom is of extreme importance in the training of the student. However, it is common for students to have some resistance to extra-class activities, due to a variety of factors, including: working hours; distance from school to home; private and family problems, among others. In this way to provide mechanisms that encourage the student to carry out these activities and that, in addition, facilitate the accomplishment of these activities is highly relevant. In this context, an alternative is the creation of a gamified tool that, through rewards, stimulates the student to carry out the activities and, in addition, allows the activities to be performed on any mobile device, facilitating access. In this context, we propose in this work the StudyPlay, a tool to support the performance of extra class activities.
\end{abstract}

Resumo. A realização de atividades extraclasse é de extrema importância na formação do estudante. Contudo, é comum que os estudantes apresentem certa resistência a realização destas atividades pelos mais diversos fatores, entre eles: horários de trabalho; distância da escola até a residência; problemas particulares e familiares, entre outros. Desta forma, prover mecanismos que incentivem o aluno a realizar estas atividades e que facilitem a realização destas, é altamente relevante. Neste contexto, uma alternativa é o desenvolvimento de uma ferramenta gamificada, que através de recompensas, estimule o estudante a realizar as atividades e que além disso, permita que as atividades sejam realizadas em qualquer dispositivo móvel, facilitando o acesso. Este trabalho propõe a ferramenta StudyPlay, uma ferramenta gamificada para suporte à realização de atividades extraclasse.

\section{Introdução}

O processo de ensino e aprendizagem é um processo contínuo que demanda empenho e dedicação do educador e do estudante. Para que este processo obtenha maior êxito, é importante que os conteúdos trabalhados em sala de aula continuem sendo exercitados pelo aluno após o término da aula. Corroborando com esta ideia da necessidade de estudos extraclasse, é conhecida a teoria de que o aluno que trabalham no turno inverso ao seus estudos possuem menos tempo para dedica-se ao curso e com isso compreendem maior dificuldade [Sousa et al. 1993].

Neste sentido, estudar de forma bem sucedida envolve um esforço integral na busca da aprendizagem e manter seus estudos em dia requer do estudante um planejamento de seu tempo, estabelecendo de antemão um plano de estudo para o dia, a semana e até mesmo para o ano letivo [Morgan and Deese 1970]. 
VII Congresso Brasileiro de Informática na Educação (CBIE 2018)

Anais do XXIX Simpósio Brasileiro de Informática na Educação (SBIE 2018)

Com a realização deste planejamento, o estudante estará organizando-se acerca dos itens mais relevantes do programa educacional e com isso poderá demandar mais tempo para as atividades as quais possui maior dificuldade ou que são considerados conteúdos chaves no plano de curso. Um exemplo clássico deste cenário são os cursos de programação de computadores, onde apenas o tempo demandado em aula para que o professor ensine o aluno não é suficiente para que o estudante desenvolva e exercite a lógica de programação, sendo necessário que o estudante desenvolva atividades extraclasse para aprofundar e fixar os conteúdos trabalhados em aula.

Contudo, o processo de ensino é um processo multidisciplinar apoiado por diversos pilares, além da continuidade do ensino, outros fatores são importantes para a formação do estudante como por exemplo a Teoria da Aprendizagem Significativa [Pelizzari et al. 2002]. Esta teoria considera que aprendizagem é dita significativa quando uma nova informação (conceito, ideia, proposição) adquire significados para o estudante através de conhecimentos prévios que o estudante já possui, estabelecendo-se uma ancoragem e um processo de evolução mutua entre o conhecimento prévio e o conhecimento adquirido.

A aprendizagem significativa não acontece apenas a retenção da estrutura do conhecimento, mas busca-se desenvolver na capacidade de transferir esse conhecimento para a sua possível utilização, em um contexto diferente daquele em que ela se concretizou. Esta ação gera autonomia frente aos saberes adquiridos e os torna mobilizáveis pelo estudante a qualquer momento e situação.

Considerando este cenário, promover estratégias que forneçam ao autonomia e estímulos ao estudante para que seu processo de aprendizagem seja continuo e que considerem seus conhecimentos prévios é de grande importância [Ausubel 2003]. Uma das estratégias que vem sendo utilizadas para estimular a realização de atividades cotidianas e que permite relacionar o conhecimento estudado, com elementos já conhecidos pelo estudante, i.e. elementos de jogos, é a gamificação (gamification) [Deterding et al. 2011]. A gamificação adiciona elementos de jogos a estas atividades, tornando-as mais atraentes, divertidas e acessíveis. Destacamos dentro dos elementos presentes em estratégias de ensino baseadas em gamificação os Rankings de pontuação e as recompensas.

Neste trabalho busca-se empregar gamificação no contexto de educação. Desta forma, o artigo propõe um sistema que permite ao professor disponibilizar atividades extraclasse e atribuir pontuações a estas atividades de acordo com critérios escolhidos por ele, por exemplo: tempo ou complexidade. Além disso, o sistema proposto considera métricas para ranqueamento dos alunos de acordo com o desempenho nas atividades, atribuindo premiações para os alunos com maior produtividade, proatividade, reatividade e interesse [Sparremberger et al. 2017].

Este trabalho está organizado da seguinte forma: na Seção 2 é apresentada a metodologia de pesquisa; na Seção 3 são apresentados os trabalhos relacionados com esta pesquisa; na Seção 4 é apresentada a pesquisa de campo que originou este trabalho; a Seção 5 apresenta o StudyPlay; na Seção 6 é apresentada a validação do StudyPlay e na Seção 7, as conclusões obtidas com este trabalho.

\section{Metodologia}

Para o desenvolvimento deste trabalho foi adotada uma metodologia estruturada que se dividiu nas seguintes fases: análise de trabalhos relacionados, pesquisa de campo, concepção e desenvolvimento do trabalho e avaliação.

- Análise de trabalhos relacionados: Realizou-se uma pesquisa utilizando a base de dados Google Scholar buscando pela string: "gamificação ou gamification e 
VII Congresso Brasileiro de Informática na Educação (CBIE 2018)

Anais do XXIX Simpósio Brasileiro de Informática na Educação (SBIE 2018)

atividades extraclasse". A partir dos artigos e resultados obtidos nesta pesquisa, foram elencados os trabalhos que se relacionam com o trabalho apresentado. Os trabalhos elencados estão descritos na Seção 3.

- Pesquisa de campo: Foi realizada uma pesquisa de campo entre alunos de cursos Técnicos em Informática, Técnico em Administração, Técnico em Redes de Computadores e Técnico em Programação de Jogos Digitais. Esta pesquisa visou captar a opinião dos alunos em relação a influência que a gamificação pode ter ou não, no empenho de estudantes na realização de atividades extraclasse. A Seção 4 apresenta os resultados desta pesquisa.

- Concepção do modelo gamificado de motivação para realização de atividades extraclasse e desenvolvimento do aplicativo StudyPlay: analisando os resultados obtidos na pesquisa de campo, foi concebido um modelo de ferramenta que poderia auxiliar os alunos no processo de realização de atividades extraclasse e motiválos para realização das mesmas. Posteriormente foi conduzido o desenvolvimento desta ferramenta das versões web e mobile.

- Validação do modelo e do aplicativo StudyPlay: Com o propósito de validar se o aplicativo projetado atingiria as expectativas dos estudantes, realizou-se a prototipação de telas que simularam a interface do aplicativo em desenvolvimento. Estas telas foram apresentadas para alguns grupos de estudantes, sendo solicitado a eles que respondessem um questionário externalizando as suas percepções a respeito do aplicativo.

\section{Análise de Trabalhos Relacionados}

A partir de uma busca efetuada na base de dados Google Scholar, foram localizados alguns trabalhos que se relacionam com o StudyPlay, sendo estes descritos nesta Seção.

[Campos et al. 2015] apresentam um trabalho que versa sobre o desenvolvimento de uma ferramenta gamificada para apoio à aprendizagem de programação no ensino superior. Esta ferramenta se diferencia do StudyPlay, visto que os objetivos do StudyPlay não são focados apenas no ensino de programação ou conhecimentos técnicos, mas também, no desenvolvimento de outras habilidades tais como engajamento e trabalho em equipe.

[Falcão 2015] propõe um sistema web gamificado para apoio ao aprendizado fazendo uso de técnicas de gamificação, como pontuação e conquistas, para estimular o interesse e a atenção dos estudantes. Este sistema se assemelha ao StudyPlay por também ter motivação focada em estimular a realização de atividades extraclasse e por fazer uso de gamificação. As principais diferenças entre os dois trabalhos são: o StudyPlay é desenvolvido seguindo a estratégia de Mobile First, tornando possível ao estudante realizar atividades via dispositivo móvel. Além disso o StudyPlay prevê a possibilidade de interação entre os estudantes através dos comentários nas atividades.

[Falcão et al. 2014], apresentam uma ferramenta de apoio à aprendizagem, que faz uso de gamificação e design de jogos, para estimular o interesse dos alunos fora da sala de aula. Este trabalho se assemelha ao StudyPlay devido aos seus objetivos, contudo neste trabalho não existe ranqueamento dos alunos e a ferramenta não está disponível em versão mobile.

[Sparremberger et al. 2017] apresenta um modelo para cálculo de proatividade, reatividade, interesse e quartil de melhor desempenho e pior desempenho para alunos de cursos presenciais e EaD. A relação deste trabalho com o StudyPlay se dá por ambos realizarem ranqueamento de alunos considerando proatividade, reatividade e interesse, contudo, o StudyPlay é uma ferramenta que suporta realização de atividades, enquanto que o Grouprofile é um framework e um monitor de métricas. 
VII Congresso Brasileiro de Informática na Educação (CBIE 2018)

Anais do XXIX Simpósio Brasileiro de Informática na Educação (SBIE 2018)

[Von Ahn 2013] fala em seu trabalho sobre a ferramenta Duolingo. Esta ferramenta faz uso de gamificação para o ensino de idiomas. A relação deste trabalho com o StudyPlay é o fato de ambos serem aplicativos para dispositivos móveis que tem como principal motivador para realização de atividades, a gamificação. Entretanto, o Duolingo não considera o suporte de um professor, que atribui pontuações para atividades e personaliza as atividades conforme o grupo de estudantes. Além disso, o Duolingo é restrito para ensino de idiomas enquanto que, o StudyPlay pode ser aplicado nas mais diversas áreas do conhecimento.

\section{Pesquisa De Campo}

Para realização da pesquisa de campo, utilizou-se um survey em formato de questionário. Este survey foi respondido por vinte e um estudantes de cursos técnicos, com idades entre dezesseis e vinte e cinco anos. Os alunos responderam perguntas objetivas a respeito do que o motiva para realizar atividades extraclasse e o que costuma impedir ou dificultar a realização dessas atividades. As perguntas efetuadas, respostas obtidas para cada pergunta e a análise das respostas são apresentadas nas próximas seções:

\subsection{Questão 01: Quando solicitada uma atividade extraclasse não avaliativa, com que frequência você costuma realiza-lá?}

Quando questionados sobre a frequência com que realizam atividades extraclasse, quando estas são solicitadas e não são avaliativas, nove dos alunos responderam que sempre realizam as atividades, seis responderam que realizam as atividades na maioria das vezes, dois em aproximadamente metade dos casos, dois e em menos da metade dos casos e um aluno respondeu raramente.

Analisando estas respostas pode-se perceber que existe uma grande parcela dos alunos que não realiza todas as atividades extraclasse solicitadas. Isto certamente impacta no aprendizado destes alunos e no andamento das atividades em sala de aula, uma vez que, se em um grupo de alunos todos ou a grande maioria buscar ampliar seus conhecimentos fora de sala de aula, em sala de aula o docente poderá aprofundar os conhecimentos curriculares ou ir além, explorando novos horizontes. Para esta análise, Levamos em consideração uma proposta denominada Just-in-Time Teaching que é uma estratégia de ensino-aprendizagem que mescla aulas presenciais, em que o estudante tem uma participação ativa, com recursos da web, que visam motivar e preparar o estudante para as aulas presenciais, bem como subsidiar os professores para que suas aulas atendam as necessidades dos estudantes[Novak et al. 1999]. Considera-se que esta grande parcela de alunos que não realiza todas as atividades que são solicitadas, pode ser motivado pela estratégia de Just-in-Time Teaching sendo possível então, ampliar o seu engajamento com o curso.

\subsection{Questão 02: Quando você não realiza a atividade, qual o principal impeditivo}

Quando questionados sobre qual o principal motivo de não realizarem todas as atividades propostas, onze dos vinte e um alunos respondentes consideram tempo o principal impeditivo. Quanto aos demais, dois alunos alegam dificuldade em estar em um local com computador acessível para realizar a atividade, quatro alunos consideram que não fazem as atividades devido ao nível de dificuldade, dois devido a falta de recompensa para realização das atividades e dois porque não consideram importante.

Considerando as respostas supradescritas, podemos perceber que mais da metade dos respondentes consideram o tempo o seu principal empecilho. Acredita-se que, ao ser disponibiliza, uma plataforma onde seja possível responder as atividades em qualquer lugar utilizando um dispositivo móvel, os problemas de tempo serão parcialmente 
VII Congresso Brasileiro de Informática na Educação (CBIE 2018)

Anais do XXIX Simpósio Brasileiro de Informática na Educação (SBIE 2018)

mitigados. Isto porque esta ferramenta poderá permitir que o estudante realize estas atividades em horários costumeiramente pouco produtivos, por exemplo, nos períodos de deslocamento. O desenvolvimento de uma solução para realização de atividades utilizando dispositivos móveis além de auxiliar os estudantes influenciados pela disponibilidade de tempo, ela também pode auxiliar os alunos que consideram o principal fator impeditivo estar em um local com computador acessível para realizar as atividades.

Por outro lado, para os alunos que não realizam as atividades devido a falta de recompensas ou pelo nível de dificuldade, se faz necessário uma ferramenta que ofereça recompensas para cada atividade realizada e que forneça feedback nas atividades para que eles saibam quando responderam de forma incorreta uma atividade e então possam corrigi-la. Este comportamento está diretamente ligado ao tipo de motivação caracterizado como Motivação Extrínseca definida como a motivação do individuo em realizar uma determinada tarefa por causa de recompensas externas a execução desta[Guimarães et al. 2004].

\subsection{Questão 03: O que te motivaria a realizar uma atividade extraclasse não avaliativa}

Para esta pergunta obtivemos as seguintes respostas: oito alunos responderam possibilidade de trabalhar em grupo, três alunos responderam recompensas, dois alunos responderam competição entre os colegas, três alunos responderam facilidade de acesso a realização da atividade, via celular por exemplo, um aluno respondeu que as atividades precisam ter uma linguagem acessível, um aluno respondeu que se motiva caso possua um local físico adequado para a realização, dois alunos responderam possuir mais tempo, um aluno respondeu que se motiva pela possibilidade de discutir com os colegas as respostas.

É possível perceber que para os estudantes é importante a interação com o grupo, a coletividade e a possibilidade de aprendizagem colaborativa. Este processo de aprendizagem colaborativa promove um maior desenvolvimento cognitivo do que estratégias que propõem exclusivamente trabalhos individuais pelos mesmos indivíduos[Moreira et al. 2016]. Com estes dados podemos concluir que uma ferramenta para apoio a realização de atividades extraclasse precisa permitir que os alunos interajam, trabalhando coletivamente e podendo discutir as suas respostas. Além disso a ferramenta precisa ter uma linguagem acessível que motive o aluno a estudar[Moreira et al. 2016].

\subsection{Questão 04: Dentre os pontos a seguir selecione os que você acredita que seriam úteis em uma ferramenta para realizar atividades extraclasse}

Para esta questão foi utilizado formato de múltipla escolha. Desta forma, os alunos puderam selecionar mais de uma opção. O Gráfico 1 resume as respostas obtidas.

No Gráfico 1 é possível perceber que as alternativas que tiveram maior adesão foram as alternativas representadas pelas barras um e sete, sendo estas respectivamente: Ranking entre a turma para eleição dos melhores alunos e Possibilidade de avançar o nível de dificuldade das tarefas pelo número de acertos. Na sequência, destaca-se a barra de número dois que representa recompensa por atividade realizada, seguida respectivamente da barra de número quatro que representa portabilidade mobile e web, barra de número cinco que representa interface acessível e barra de número seis que representa possibilidade de criação de avatar para representação do aluno.

Estas respostas permitiram identificar que todas as características apontadas foram consideradas importantes pelos alunos, uma vez que todas elas foram selecionadas por um ou mais alunos, destacando-se: Ranking entre a turma para eleição dos melhores alunos e Possibilidade de avançar o nível de dificuldade das tarefas pelo número de acerto 
VII Congresso Brasileiro de Informática na Educação (CBIE 2018)

Anais do XXIX Simpósio Brasileiro de Informática na Educação (SBIE 2018)

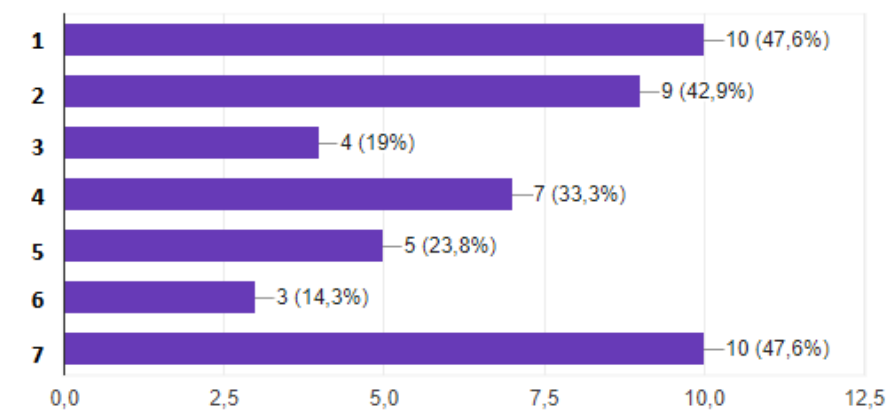

Figura 1. Características importantes ferramenta para atividades extraclasse

\section{Concepção e Desenvolvimento do StudyPlay}

Analisando as respostas obtidas na pesquisa de campo, pode-se concluir que uma ferramenta para incentivo a realização de atividades extraclasse precisa possuir as seguintes características:

1. Permitir a realização das atividades via celular e via web;

2. Realizar ranqueamento de alunos de acordo com o desempenho

3. Premiar alunos com maior produtividade, reatividade e interesse

4. Possuir uma interface amigável e acessível

5. Permitir realizar as atividades de forma offline e apenas enviar de forma online

6. Feedback rápido

7. Gabarito disponível

8. Possibilidade de interação com os colegas

Desta forma, propõe-se o aplicativo StudyPlay, um aplicativo que contempla todas as características supracitas sendo personalizável para se adaptar a qualquer turma ou curso. Para atender a esta adaptabilidade o StudyPlay compreende dois tipos de atividades, sendo eles: atividade objetiva e atividade descritiva. Na categoria atividade objetiva o aluno irá receber feedback instantâneo para as suas respostas, e na categoria descritiva as respostas são enviadas para o professor, para que este efetue a correção e atribua certo ou errado.

O fluxo de trabalho no StudyPlay, conforme supracitado contempla três papéis principais sendo eles: o professor, o estudante e o sistema. As atividades realizadas por cada um podem ser visualizadas na Figura2

Cadastra Atividades

Figura 2. Papéis StudyPlay

\subsection{Métricas utilizadas para ranqueamento e premiação destaque proatividade, reatividade e interesse}

Um dos principais pontos utilizados pelo StudyPlay no que diz respeito a gamificação é o ranqueamento dos alunos de acordo com o desempenho nas atividades e os índices de 
VII Congresso Brasileiro de Informática na Educação (CBIE 2018)

Anais do XXIX Simpósio Brasileiro de Informática na Educação (SBIE 2018)

proatividade, reatividade e interesse.

Para o ranqueamento geral considerou-se apenas a pontuação das atividades, sendo feito o somatório dos pontos acumulados pelo aluno e classificado em ordem decrescente. Para alunos com a mesma pontuação, utiliza-se como critério de desempate o índice PRI, estabelecido pelo modelo Grouprofile [Sparremberger et al. 2017], que considera os valores de proatividade, reatividade e interesse. De igual forma, para atribuição dos destaques proatividade, reatividade e interesse utilizou-se uma adaptação do cálculo destes valores também utilizado pelo modelo Grouprofile.

Para proatividade, reatividade e interesse a periodicidade em que serão atribuídos os destaques será definido pelo professor responsável pela turma ou grupo. Já o ranking de pontuação é atualizado continuamente sempre que o aluno realiza alguma atividade.

\subsubsection{Métrica StudyPlay para cálculo de Proatividade}

Para a definição das métricas de proatividade seguiu-se a mesma linha de pontuação utilizada pelo modelo Grouprofile, sendo feitas algumas adaptações para o contexto do StudyPlay. Desta forma ficam definidos os seguintes parâmetros para cálculo de proatividade.

- Número de iterações com o aplicativo StudyPlay maior ou igual a média da turma3 pontos;

- Periodicidade de acessos ao aplicativo - 3 pontos

Alunos que efetuam acesso apenas no período de até 24 horas antes do período limite para realização das atividades, contabilizam 1 ponto.

Alunos que acessam o ambiente continuamente, 2 pontos.

Alunos que acessam continuamente e no período de até 24 horas após o professor ter lançado uma nova atividade, 3 pontos.

- Realização das atividades - 3 pontos

Alunos que fazem as atividades no período das primeiras 24 horas prazo, 3 pontos.

Alunos que fazem as atividades na primeira metade do prazo, 2 pontos.

Alunos que fazem as atividades na segunda metade do prazo, 1 ponto.

- Número de acessos - 0,5 Pontos

Alunos com número de acesso superior a média, 0,5 ponto

- Cadastro Completo - 0,5 Ponto

Alunos com cadastro completo, 0,5 ponto

\subsubsection{Métrica StudyPlay para cálculo de Reatividade}

A definição das métricas de reatividade também foi baseada no modelo Grouprofile, sendo feitas as devidas adaptações e que culminaram nas seguintes métricas:

- Realização de atividades - 4 pontos: O cálculo desta pontuação será feito da seguinte maneira: total de atividades efetuadas no prazo(EP), divididas pelo total de atividades solicitas(AS) e multiplicado por quatro.

- Número de acessos (com realização de atividades) ao StudyPlay- 2 pontos

Acima da média do grupo: 2 pontos;

Acima da metade média do grupo: 1 ponto;

Maior que zero e até a média do grupo: 0,5 pontos.

- Número de comentários em atividades - 2 pontos

Acima da média do grupo: 1 ponto;

Maior que zero e até a média do grupo: 0.5 ponto; 
VII Congresso Brasileiro de Informática na Educação (CBIE 2018)

Anais do XXIX Simpósio Brasileiro de Informática na Educação (SBIE 2018)

- Número de respostas a comentários dos colegas nas atividades- 2 pontos:

Acima da média do grupo: 1 ponto;

Maior que zero e até a média do grupo: 0,5 ponto.

\subsubsection{Métrica StudyPlay para cálculo de Interesse}

Os cálculos de interesse no aplicativo StudyPlay, são calculados da seguinte maneira:

- Aluno com maior constância de acessos ao aplicativo - 2 pontos

- Aluno com maior tempo de permanência ativo no aplicativo - 2 pontos

- Aluno com o maior número de atividades respondidas consecutivamente (no mesmo acesso) - 3 pontos

- Aluno que mais vezes refez as atividades - 3 pontos

Para todos os itens estipulados para cálculo de interesse, em caso de empate, a pontuação é atribuída a todos. No caso de empate na pontuação total , todos os empatados recebem premiação de destaque.

\subsubsection{Cálculo de PRI StudyPlay}

O índice PRI utilizado como critério de desempate para definição do ranqueamento dos alunos é dado pela seguinte equação [Sparremberger et al. 2017].

$$
\mathbf{P R I}=(\text { Proatividade } * 0.4)+(\text { Reatividade } * 0.3)+(\text { Interesse } * 0.3)
$$

\section{Avaliação e Resultados}

A avaliação deste trabalho baseou-se nas funcionalidades definidas para o StudyPlay, e teve como objetivo principal identificar se o aplicativo iria de fato atender as expectativas externalizadas pelos estudantes na pesquisa de campo. Apresentamos nesta Seção uma validação da proposta de aplicativo bem como da metodologia gamificada de estímulo a realização das atividades extraclasse.

Para realização desta validação consideramos duas etapas, sendo elas: demonstração dos protótipos e do funcionamento das telas prototipadas e survey em formato de questionário.

\subsection{Demonstração Das Telas e Funcionalidades}

Para esta etapa da validação, foram apresentados aos alunos as telas do aplicativo. Através destas telas os alunos puderam conhecer o funcionamento o layout do aplicativo e analisar se este traria benefícios a eles ou não. Para esta demonstração, foram priorizadas as telas com as quais os alunos tem contato, sendo elas as seguintes: tela onde os estudantes respondem as perguntas; tela onde os estudantes recebem feedback das respostas; e a tela onde é exibido o ranqueamento dos alunos com melhor desempenho e os destaques proatividade, reatividade e interesse. As telas do aplicativo podem ser visualizados na Figura3.

Na Figura 3 pode ser visualizado no ranqueamento alguns personagens fictícios. Estes personagens representam apelidos e avatares escolhidos por alunos para representálos no ranking de pontuação. Além de ter contato com as telas, neste momento foi explicado aos alunos o funcionamento das funcionalidades principais do sistema, por exemplo: sistema de pontuação; correção e feedback automatizado; atribuição de destaque proatividade, destaque reatividade e destaque interesse. 
VII Congresso Brasileiro de Informática na Educação (CBIE 2018)

Anais do XXIX Simpósio Brasileiro de Informática na Educação (SBIE 2018)

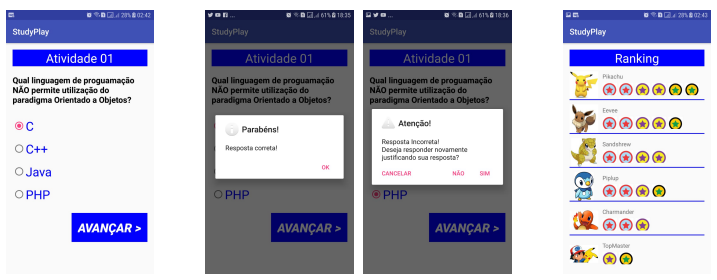

Figura 3. Protótipos apresentados para validação: questão e validação de resposta correta

\subsection{Realização De Survey em Formato de Questionário}

Após conhecerem o protótipo das telas e estarem cientes da sua funcionalidade os alunos responderam a um questionário onde puderam registrar a sua percepção a respeito do aplicativo. As perguntas efetuadas foram as seguintes:

1. Caso este aplicativo estivesse disponível para você, o que você faria?

2. De forma geral o que você achou da ideia de desenvolvimento deste aplicativo?

3. Você utilizaria o aplicativo?

Esta pesquisa contou com a resposta de vinte e dois estudantes de nível técnico de curso Técnico em Informática. Para a primeira pergunta que versou sobre qual seria a posicionamento do aluno caso o aplicativo já estivesse disponível para uso, doze estudantes consideraram que se esforçariam mais para fazer as atividades; seis estudantes responderam que aumentaria o interesse; três estudantes responderam que não seriam influenciados e um que utilizaria apenas se fossem atribuídas notas para as atividades.

Analisando estas respostas pode-se perceber que a maioria dos estudantes acredita que será positivamente influenciado quando o aplicativo estiver disponível para o uso, aumentando o interesse e por consequência depreendendo um maior esforço. Acredita-se que com isso que o aplicativo irá agregar valor ajudando a reduzir o número de alunos que não realiza as atividades extraclasse propostas ou realiza parcialmente.

Para a segunda pergunta, cujo foco foi descobrir qual a percepção geral dos alunos sobre a iniciativa da criação de uma ferramenta gamificada para realização das atividades, obtivemos as seguintes respostas: quatorze alunos consideram a iniciativa válida, e acreditam que vai estimular a realização de atividades; sete alunos acreditam que com o aplicativo vão aprender mais e aproveitar melhor o curso e um aluno considera que irá se sentir reprimido pelo esquema de ranqueamento.

Acredita-se, baseado nestas respostas, que o aplicativo pode influenciar positivamente a grande maioria dos alunos para melhorar o aprendizado e o aproveitamento do curso. Em relação ao aluno que respondeu que se sentiria reprimido, é importante enfatizar que o ranqueamento dos estudantes será feito utilizando apelidos, e desta forma os colegas não terão acesso ao nome dos alunos ranqueados, exceto em situações onde o aluno deseja externalizar sua pontuação e por isso atribui o próprio nome ao apelido.

A terceira e última pergunta diz respeito a utilização do aplicativo. Onde os estudantes responderam que utilizariam o aplicativo; seis estudantes responderam que utilizariam mas sem intuito de competição, três estudantes utilizariam buscando competir com os colegas e dois estudantes responderam que não utilizariam, sendo um porque consumiria tempo e um porque acredita que não teria aprendizado com o aplicativo.

Identificou-se nestas respostas, uma grande adesão e motivação para utilização do aplicativo. Isto viabiliza e justifica a sua criação e a concepção do modelo gamificado de incentivo a realização de atividades extraclasse. 
VII Congresso Brasileiro de Informática na Educação (CBIE 2018)

Anais do XXIX Simpósio Brasileiro de Informática na Educação (SBIE 2018)

\section{Conclusões}

Após a realização da pesquisa de campo, concepção do modelo, desenvolvimento do aplicativo e validação, foi possível identificar que o modelo e a ferramenta StudyPlay poderão trazer benefícios para os estudantes das instituições que optarem pela sua utilização. Isto é devido a utilização desta ferramenta, ter sido relacionada pelos alunos como um possível fator motivador e gerador de conhecimento. O aplicativo StudyPlay ainda está em fase de testes e em breve deverá ser disponibilizado para utilização dos estudantes.

\section{Referências}

Ausubel, D. P. (2003). Aquisição e retenção de conhecimentos: uma perspectiva cognitiva. Lisboa: Plátano, 1.

Campos, A., Gardiman, R., and Madeira, C. (2015). Uma ferramenta gamificada de apoio à disciplina introdutória de programação. $23^{\circ}$ WEI-WORKHOP SOBRE EDUCAÇÃO EM COMPUTAÇÃO.

Deterding, S., Sicart, M., Nacke, L., O'Hara, K., and Dixon, D. (2011). Gamification. using game-design elements in non-gaming contexts. In CHI'11 extended abstracts on human factors in computing systems, pages 2425-2428. ACM.

Falcão, A. P. (2015). Gamersbook: sistema gamificado de apoio ao aprendizado. B.S. thesis, Universidade Tecnológica Federal do Paraná.

Falcão, A. P., Leite, M. D., and Tenório, M. M. (2014). Ferramenta de apoio ao ensino presencial utilizando gamificação e design de jogos. In Brazilian Symposium on Computers in Education (Simpósio Brasileiro de Informática na Educação-SBIE), volume 25, page 526 .

Guimarães, S. É. R., Boruchovitch, E., et al. (2004). O estilo motivacional do professor e a motivação intrínseca dos estudantes: uma perspectiva da teoria da autodeterminação. Psicologia: reflexão e crítica, 17(2):143-150.

Moreira, F., Ferreira, M. J., Pereira, C. S., and Durão, N. (2016). Collaborative learning supported by mobile devices: A case study in portuguese high education institutions. In New Advances in Information Systems and Technologies, pages 157-167. Springer.

Morgan, C. T. and Deese, J. (1970). Como Estudar. Livraria Freitas Bastos, 1th edition.

Novak, G., Patterson, E., Gavrin, A., and Christian, W. (1999). Just-in-time teaching: Blending active learning with web technology prentice hall. Pretentice Hall.

Pelizzari, A., Kriegl, M. d. L., Baron, M. P., Finck, N. T. L., and Dorocinski, S. I. (2002). Teoria da aprendizagem significativa segundo ausubel. revista PEC, 2(1):37-42.

Sousa, J., Bianchetti, L., MOTA, L., ALMEIDA, V. d., and INEP (1993). Estudo do aluno universitário para a construção de um projeto pedagógico. MEC/INEP. Série Documental em Relatos de Pesquisa, 4.

Sparremberger, A. S., Barbosa, J., and Gluz, J. (2017). Grouprofile: Um modelo de gerenciamento de perfis de grupos de aprendizes. In Brazilian Symposium on Computers in Education (Simpósio Brasileiro de Informática na Educação-SBIE), volume 28, page 1687.

Von Ahn, L. (2013). Duolingo: learn a language for free while helping to translate the web. In Proceedings of the 2013 international conference on Intelligent user interfaces, pages 1-2. ACM. 